\title{
SOME NEW INTEGRAL INEQUALITIES ON TIME SCALES
}

\author{
Feifei Du, Wei Hu, Lynn ERBe And Allan Peterson
}

Abstract. In this paper, we establish some generalizations of inequalities on time scales, which have appeared in different articles. The inequalities that we will derive from our results when $g(t)=t$ are essentially new.

Mathematics subject classification (2010): 39A12, 39A70.

Keywords and phrases: Integral inequalities, time scales, Feng-Qi inequality.

\section{REFERENCES}

[1] C. D. Ahlbrandt and C. Morian, Partial differential equations on time scales, J. Comput. Appl. Math. 141, (2002), 35-55.

[2] R. P. Agarwal, D. O'Regan And S. H. SAKer, Hardy Type Inequalities on Time Scales, Springer, Switzerland, 2016.

[3] E. AKIN, Cauchy functions for dynamic equations on a measure chain, Math. Anal. Appl. 267, (2002), $97-115$.

[4] M. Bohner And A. Peterson, Dynamic Equations on Time Scales: An Introduction with Applications, Birkhäuser, Boston, 2001.

[5] M. Bohner And A. Peterson, Advances in Dynamic Equations on Time Scales, Birkhäuser, Boston, 2003.

[6] K. Brahim, N. Bettaibi and M. Sellami, On some Feng-Qi type q-integral inequalities, J. Inequal. Pure Appl. Math. 9, 2(2008), 1-7.

[7] T. FAyYaz, N. IRShad, A. Khan, G. Rahman AND G. RoQIA, Generalized integral inequalities on time scales, J. Inequal. Appl. 235, (2016), 1-12.

[8] S. Hilger, Ein Maßkettenkalkül mit Anwendung auf Zentrumsmannig-faltigkeiten, $\mathrm{PhD}$ thesis, Universität Würzburg, 1988.

[9] B. KARPUZ, Unbounded oscillation of higher-order nonlinear delay dynamic equations of neutral type with oscillating coefficients, Electron. J. Qual. Theory Differ. Equ. 34, (2009), 1-14.

[10] B. KARPUZ, Volterra theory on time scales, Results. Math. 65, (2014), 263-292.

[11] S. Keller, Asymptotisches Verhalten invarianter Faserbündel bei Diskretisierung und Mittelwertbildung im Rahmen der Analysis auf Zeitskalen, PhD thesis, Universität Augsburg, 1999.

[12] Y. Miao And F. QI, Several q-integral inequalities, J. Math. Inequal. 3, 1(2009), 115-121.

[13] C. PÖTZSCHE, Chain rule and invariance principle on measure chains, J. Comput. Appl. Math. 141, (2002), 249-254.

[14] F. QI, A. J. LI, W. Z. ZhaO, D. W. NiU AND J. CAO, Extensions of several integral inequalities, J. Inequal. Pure Appl. Math. 7, 3(2006), 1-4.

[15] F. QI, Several integral inequalities, J. Inequal. Pure Appl. Math. 1, 2(2000), 1-3.

[16] M. R. Segi Rahmat, On some $(q, h)$-analogues of integral inequalities on discrete time scales, Comput. Math. Appl. 62, 2(2000), 1790-1797.

[17] W. Sulaiman, New Types of Q-Integral Inequalities, Adv. Pure. Math. 1, (2011), 77-80.

[18] W. Sulaiman, A Study on New q-Integral Inequalities, Appl. Math. 1, (2011), 465-469.

[19] W. Sulaiman, Several Ideas on Some Integral Inequalities, Adv. Pure. Math. 1, (2011), 63-66.

[20] L. Yin, Q. Luo AND F. QI, Several integral inequalities on time scales, J. Math. Inequal. 6, (2012), 419-429. 